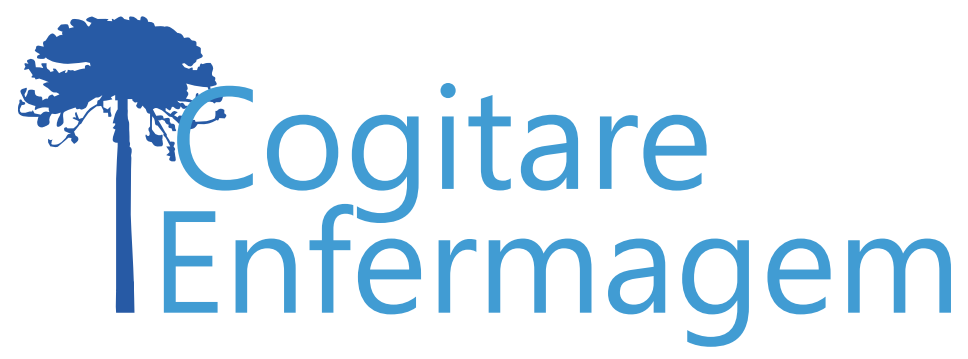

ARTIGO ORIGINAL

\title{
INSTRUMENTOS DE AVALIAÇÃO DA LITERACIA EM SAÚDE ACERCA DO TABACO: TRADUÇÃO E ADAPTAÇÃO PARA PORTUGUÊS EUROPEU
}

\section{INSTRUMENTS FOR ASSESSING TOBACCO-RELATED HEALTH LITERACY: TRANSLATION AND ADAPTATION TO EUROPEAN PORTUGUESE}

\author{
Daniela Lourenço Pinto ${ }^{1}$ (1) \\ Heidi Parisod ${ }^{2}$ (1) \\ Johanna Nyman² (i) \\ Tereza Maria Mendes Dinis de Andrade Barroso ${ }^{1}$ (])
}

\begin{abstract}
Objectives: To translate and culturally adapt to European Portuguese the tobacco-related health literacy assessment instruments "Attitudes Towards Tobacco Use," "Tobacco-Use Motives," "Motivation to Decline Tobacco Use in the Future," "Smoking Outcome Expectation Scale," and "Anti-Smoking Self-Efficacy Scale"; and to conduct the preliminary validation of the translated versions. Methodology: Translation and cross-cultural adaptation in accordance with the Institute for Work \& Health recommendations and preliminary validation study using a sample of 144 adolescents from two public schools in central Portugal in April and May 2019. Results: Snus-related items were removed due to their lack of relevance in the Portuguese context. Thus, two instruments consisted of only one item each. The European Portuguese versions of the instruments presented Cronbach's alpha scores of $0.799,0.673,0.905$, and 0.890 . Conclusion: The present study contributed to developing European Portuguese versions of instruments for assessing tobacco-related health literacy among adolescents.
\end{abstract}

DESCRIPTORS: Validation Study; Scales; Health Literacy; Tobacco; Adolescents.

COMO REFERENCIAR ESTE ARTIGO:

Pinto DL, Parisod H, Nyman J, Barroso TMMD de A. Instrumentos de avaliação da literacia em saúde acerca do tabaco: tradução e adaptação para português europeu. Cogit. Enferm. [Internet]. 2021 [acesso em "colocar data de acesso, dia, mês abreviado e ano"]; 26. Disponível em: http://dx.doi.org/10.5380/ce.v26i0.80555.

'Unidade de Investigação em Ciências da Saúde: Enfermagem, Escola Superior de Enfermagem de Coimbra. Coimbra, Portugal.

${ }^{2}$ University of Turku, Department of Nursing Science. Turku, Finlândia. 
A literacia em saúde permite capacitar as crianças e adolescentes para se tornarem mais informados e críticos acerca das futuras escolhas em relação à própria saúde ${ }^{(1)}$. Alguns estudos $^{(2)}$ referem que é mais provável que adolescentes com baixos níveis de literacia adotem condutas de risco, como o consumo de substâncias, pelo que é de extrema importância desenvolver intervenções e instrumentos de avaliação da literacia em saúde adequados a crianças e adolescentes, e específicos, por exemplo, para as questões do tabaco.

Neste sentido, foi desenvolvido o Fume ${ }^{(3)}$, um mobile health game para crianças e adolescentes para melhorar a literacia em saúde acerca do tabaco, desenvolvido na Universidade de Turku, Finlândia, e publicado em 2016 em finlandês, sueco e inglês, tendose considerado relevante o desenvolvimento da versão portuguesa.

Para a avaliação do efeito da versão portuguesa, e tendo em conta que não foram encontrados instrumentos de avaliação da literacia em saúde acerca do tabaco ou da autoeficácia antitabaco desenvolvidos em Portugal ou adaptados para a população portuguesa, e especificamente para adolescentes, considerou-se pertinente a utilização dos mesmos instrumentos utilizados no estudo de avaliação do efeito do Fume, o que permitiria uma comparação dos resultados com a versão original. Assim, tornou-se necessária a tradução e adaptação dos instrumentos de avaliação para português europeu.

Desta forma, os objetivos deste estudo foram: (i) traduzir e adaptar culturalmente para português europeu os instrumentos de avaliação Attitudes Towards Tobacco Use, Tobbaco-Use Motives e Motivation to Decline Tobbaco Use in the Future(4), e as escalas Anti-Smoking Self-Efficacy Scale (ASSES) e Smoking Outcome Expectation Scale (SOES)(5); (ii) realizar uma validação preliminar das versões traduzidas e adaptadas para português europeu dos instrumentos.

\section{MÉTODO}

O presente estudo deriva de uma parceria entre a Escola Superior de Enfermagem de Coimbra e a Universidade de Turku, na Finlândia, sendo parte de uma dissertação de mestrado.

É um estudo metodológico, dividido em duas etapas: tradução e adaptação transcultural para português europeu e validação preliminar das versões traduzidas dos instrumentos Attitudes Towards Tobacco Use, Tobbaco-Use Motives e Motivation to Decline Tobbaco Use in the Future, desenvolvidos por Parisod e colaboradores ${ }^{(4)}$, e ASSES e SOES, de Chen e colaboradores ${ }^{(5)}$.

Os instrumentos Attitudes Towards Tobacco Use, Tobbaco-Use Motives e Motivation to Decline Tobbaco Use in the Future foram desenvolvidos no âmbito do estudo de avaliação do efeito do jogo Fume, com base num estudo prévio, cujo objetivo foi identificar os determinantes da literacia em saúde relacionada com o tabaco, na perspetiva dos adolescentes ${ }^{(6)}$.

O Attitudes Towards Tobacco Use inclui duas subescalas, cada uma com um item mensurado de um (very stupid) a quatro (very ok), tem como objetivo avaliar as atitudes dos adolescentes face ao ato de fumar cigarros e ao uso de snus e a pontuação varia entre um e quatro pontos, correspondendo um à atitude mais negativa perante fumar cigarros/ consumir snus ${ }^{(4)}$. 
O Tobbaco-Use Motives inclui duas subescalas, uma relativa aos motivos de caráter social que podem levar os adolescentes a fumar cigarros e outra relativa ao consumo de snus. Ambas apresentam três itens cada, cada item avaliado de um (not at all) a quatro pontos (surely), e a pontuação pode variar entre três e 12 pontos, sendo que uma pontuação de três representa menos motivos para fumar cigarros/consumir snus ${ }^{(4)}$.

O Motivation to Decline Tobacco Use in the Future inclui duas subescalas relativas à motivação para recusar tabaco no futuro, uma para o consumo de cigarros e outra para o uso de snus. Cada subescala contém um item, cuja pontuação varia de um (not at all) a quatro pontos (surely); uma pontuação total de um refere-se à mais alta motivação para recusar fumar cigarros ou consumir snus no futuro(4).

Os instrumentos ASSES e SOES foram desenvolvidos em 2015(5), com o objetivo de desenvolver escalas apropriadas para mensurar as expetativas dos pré-adolescentes sobre as consequências (outcomes) de fumar, bem como a sua autoeficácia antitabaco.

Constituída por 15 itens avaliados de um (cannot do at all) a quatro (certainly can do), a ASSES é uma escala cuja pontuação total pode variar entre 15 e 60 pontos, sendo o nível de autoeficácia para adotar comportamentos antitabaco tanto mais elevado quanto mais próximo de 60 a pontuação final se aproximar ${ }^{(4)}$.

A SOES permite avaliar, através de uma lista de seis itens avaliados de um (strongly disagree) a quatro (strongly agree), as expectativas dos adolescentes relativamente às consequências de fumar. Encontra-se dividida em duas subescalas, a Positive Smoking Outcome Expectation Scale (POS-SOES) e a Negative Smoking Outcome Expectation Scale (NEG-SOES), cada uma com três itens. A POS-SOES, cuja pontuação varia entre três e 12 pontos, permite avaliar a existência de sentimentos positivos em relação ao tabaco, e uma pontuação total elevada indica um alto nível de concordância com a ideia de que fumar transmite sentimentos de maturidade, estar na moda e de coragem ${ }^{(4-5)}$. A NEG-SOES, cuja pontuação varia também de três a 12 pontos, permite avaliar a existência de sentimentos negativos associados ao ato de fumar, representando uma pontuação total elevada um alto nível de concordância com as afirmações que incluem alguns efeitos adversos do tabaco ${ }^{(4-5)}$.

Para a primeira etapa, seguiram-se as recomendações do Institute for Work and Health ${ }^{(7)}$. As fases deste processo são(7): Fase I - tradução inicial do instrumento, do idioma de origem para o idioma pretendido, por dois tradutores com perfis diferentes: um com conhecimentos sobre os conceitos e outro que não domine a temática; Fase II - síntese das duas traduções resultantes da fase I, elaborando-se uma versão de tradução conjunta e resolvendo-se as possíveis discrepâncias existentes; Fase III - retrotradução da versão conjunta elaborada na fase II, para perceber se esta reflete o mesmo conteúdo que a versão original, efetuada por dois tradutores que não tenham conhecimento da versão original e cuja língua materna seja a do idioma original do instrumento; Fase IV - comissão de peritos multidisciplinar, com o objetivo de considerar todas as versões do instrumento elaboradas e desenvolver uma versão pré-final para a realização do pré-teste de campo; e Fase $V$ - fase de teste da versão pré-final do instrumento, na qual esta é aplicada a pessoas que se enquadram na população-alvo.

A segunda etapa consistiu na validação preliminar das versões traduzidas para português europeu dos instrumentos, através da análise da consistência interna, pelo alfa de Cronbach (a), com base na classificação de Pestana e Gageiro( ${ }^{(8)}$ : muito boa (se $a \geq 0,9$ ), boa (se $0,8 \leq \alpha<0,9$ ), razoável (se $0,7 \leq \alpha<0,8)$, fraca (se $0,6 \leq \alpha<0,7$ ) ou inadmissível (se $\alpha<0,6$ ). Foi também calculada a correlação item-total corrigida, que avalia a correlação entre cada item e o total da escala quando este item é omitido, sendo esperado que esta correlação seja superior a $0,3^{(9)}$. Calculou-se ainda o alfa de Cronbach se o item for excluído(8), o qual foi comparado com o alfa de Cronbach total da escala. Recorreu-se à análise estatística, através do software IBM SPSS Statistics, versão 25.0.

O presente estudo decorreu no âmbito do desenvolvimento do estudo de avaliação 
do efeito da versão portuguesa de um jogo de saúde dirigido a adolescentes, o No Fume.

Devido à dificuldade em reunir uma amostra significativa da população específica para a qual os instrumentos foram desenvolvidos, recorreu-se aos momentos de avaliação do efeito da versão portuguesa do jogo Fume para recolher os dados para a validação preliminar das versões em português europeu dos instrumentos. Assim, as versões traduzidas dos instrumentos foram aplicadas em dois momentos, com intervalo de 2 semanas, a uma amostra, selecionada por conveniência, constituída por adolescentes entre 10 e 13 anos.

A coleta de dados ocorreu em abril e maio de 2019, em duas escolas públicas do segundo e terceiro ciclo do ensino básico da região centro de Portugal.

Para garantir o cumprimento de todos os pressupostos éticos e formais, o estudo foi submetido à Comissão de Ética da Unidade de Investigação em Ciências da Saúde: Enfermagem, da Escola Superior de Enfermagem de Coimbra, tendo-se obtido parecer favorável (P521-09/2018). Solicitou-se o consentimento dos autores originais dos instrumentos, tendo estes formalizado a sua autorização, por escrito, através de e-mail.

Para proteger os adolescentes que participaram no estudo, foram garantidos ao longo de todo o processo o direito à autodeterminação, anonimato e confidencialidade dos dados, tendo sido preenchido um consentimento informado pelos adolescentes e respectivos encarregados de educação.

\section{RESULTADOS}

\section{Tradução e Adaptação Transcultural}

Numa primeira fase, os instrumentos foram traduzidos para português europeu por dois tradutores. Posteriormente, realizou-se uma síntese das duas e elaborou-se uma tradução conjunta. Em seguida, foi realizada a tradução da versão conjunta para o idioma original (inglês). As fases anteriormente descritas decorreram sem discrepâncias significativas, chegando-se facilmente a um consenso sobre os termos a serem utilizados nas versões finais de cada uma. Elaborou-se, posteriormente, a versão pré-final através da revisão por um grupo constituído por três profissionais especialistas em enfermagem de saúde mental e psiquiatria.

Os instrumentos Attitudes Towards Tobacco Use, Tobacco-Use Motives e Motivation to Decline Tobacco Use in the Future foram desenvolvidos com base na realidade cultural relacionada com o tabaco dos adolescentes da Finlândia ${ }^{(4)}$, pelo que, para além de itens referentes a cigarros convencionais, incluem as mesmas questões também referentes ao snus. Assim, para estes instrumentos foram retirados todos os itens referentes a snus, uma vez que se considerou que este conteúdo não apresentava relevância para o contexto português.

A escala Tobacco-Use Motives foi traduzida sem discrepâncias entre as traduções e resultou na versão em português europeu Motivos para Uso de Tabaco. Para os instrumentos Attitudes Towards Tobacco Use e Motivation to Decline Tobacco Use in the Future, os quais continham apenas dois itens, um relativo aos cigarros e outro relativo ao snus, a retirada dos itens específicos do snus conduziu a que as versões traduzidas ficassem constituídas por apenas um item cada. Assim, na sua versão em português europeu, estas passam a ser designadas não como escalas mas como itens: o item Atitudes face ao Uso de Tabaco e o item Motivação para Recusar a Utilização de Tabaco no Futuro.

A SOES, na sua versão em português europeu, passou a ser designada Escala de Expetativas sobre Fumar, e as subescalas POS-SOES e NEG-SOES passaram a designar-se, em português europeu, Escala de Expetativas Positivas sobre Fumar e Escala de Expetativas 
Negativas sobre Fumar, respectivamente. Foram mantidos os seis itens originais da SOES, os três primeiros pertencentes à Escala de Expectativas Positivas sobre Fumar e os três últimos pertencentes à Escala de Expectativas Negativas sobre Fumar, tal como nas versões originais.

A ASSES foi traduzida sem discrepâncias e, na sua versão em português europeu, passou a ser designada Escala de Autoeficácia Antitabaco.

\section{Validação Preliminar}

Para a realização do pré-teste dos instrumentos e validação preliminar, a amostra (Tabela 1), selecionada por conveniência, foi constituída por 144 adolescentes que frequentavam o sexto ano de escolaridade no ano letivo 2018/2019, em agrupamentos de escolas públicas da região centro de Portugal.

Tabela 1 - Breve caracterização da amostra do estudo preliminar de validação dos instrumentos ( $n=144)$. Coimbra, Portugal, 2021

\begin{tabular}{llc} 
Variável & & $\mathbf{n}(\%)$ \\
\hline \multirow{2}{*}{ Gênero } & Feminino & $84(58,3)$ \\
\cline { 2 - 3 } & Masculino & $60(41,7)$ \\
\cline { 2 - 3 } & 10 anos & $2(1,4)$ \\
\cline { 2 - 3 } & 11 anos & $83(57,6)$ \\
\cline { 2 - 3 } Nacionalidade & 12 anos & $54(37,5)$ \\
\cline { 2 - 3 } & Angolana & $5(3,5)$ \\
\cline { 2 - 3 } & Brasileira & $1(1,7)$ \\
\cline { 2 - 3 } & Luso-francesa & $1(0,9)$ \\
\cline { 2 - 3 } & Portuguesa & $132(91,7)$ \\
\cline { 2 - 3 } & Síria & $1(0,7)$ \\
\cline { 2 - 3 } & Venezuelana & $2(1,4)$
\end{tabular}

Fonte: Autores (2021)

A análise da consistência interna, através do alfa de Cronbach, da análise da correlação item-total corrigida e do alfa de Cronbach se o item for excluído, foram realizadas em dois momentos para os instrumentos Motivos para Uso de Tabaco, Escala de Expectativas sobre Fumar e Escala de Autoeficácia Antitabaco. Para os itens Atitudes face ao Uso de Tabaco e Motivação para Recusar Utilização de Tabaco no Futuro, uma vez que apenas são constituídas por um item cada, não foi possível o cálculo do alfa de Cronbach e restantes análises.

O instrumento Escala de Motivos para Uso de Tabaco, no primeiro momento, apresentou um alfa de Cronbach de 0,697 (Quadro 1). A média dos itens variou entre 1,15 (item um) e 1,20 (item três). No segundo momento, o valor do alfa de Cronbach foi 0,799. A média dos itens variou entre 1,16 (item dois) e 1,22 (item três). No segundo momento, caso o item três fosse excluído, o alfa de Cronbach aumentaria de 0,799 para 0,824 (Quadro 
1). Não se verificaram valores inferiores a 0,3 no que diz respeito à correlação item-total corrigido, sendo todos os valores superiores a 0,490.

Quadro 1 - Estatísticas de Item e Item-Total para a Escala de Motivos para Uso de Tabaco, no $1^{\circ}$ Momento e no $2^{\circ}$ Momento. Coimbra, Portugal, 2021

\begin{tabular}{|c|c|c|c|c|c|}
\hline \multirow[b]{2}{*}{ Avaliação } & \multirow[b]{2}{*}{ Item } & \multicolumn{2}{|c|}{ Estatísticas de Item } & \multicolumn{2}{|c|}{ Estatísticas de Item-Total } \\
\hline & & Média & $\begin{array}{l}\text { Desvio- } \\
\text { padrão }\end{array}$ & $\begin{array}{c}\text { Correlação } \\
\text { de item total } \\
\text { corrigida } \\
\end{array}$ & $\begin{array}{c}\text { Alfa de } \\
\text { Cronbach se o } \\
\text { item for excluído }\end{array}$ \\
\hline \multirow[t]{4}{*}{$1^{\circ}$ Momento } & Item 1 & 1,15 & 0,418 & 0,565 & 0,569 \\
\hline & Item 2 & 1,17 & 0,532 & 0,51 & 0,613 \\
\hline & Item 3 & 1,2 & 0,54 & 0,49 & 0,643 \\
\hline & \multicolumn{5}{|c|}{ Alfa de Cronbach Total $=0,697$} \\
\hline \multirow[t]{4}{*}{$2^{\circ}$ Momento } & Item 1 & 1,17 & 0,473 & 0,698 & 0,67 \\
\hline & Item 2 & 1,16 & 0,468 & 0,685 & 0,685 \\
\hline & Item 3 & 1,22 & 0,531 & 0,56 & 0,824 \\
\hline & \multicolumn{5}{|c|}{ Alfa de Cronbach Total $=0,799$} \\
\hline
\end{tabular}

Fonte: Autores (2021)

A Escala de Expectativas Positivas sobre Fumar apresentou, no primeiro momento, um alfa de Cronbach de 0,595 (Quadro 2). A média dos itens variou entre 1,33 (item um) e 1,78 (item dois). $O$ alfa de Cronbach no segundo momento foi de 0,673. A média dos itens variou entre 1,30 (item um) e 1,70 (item dois). Verifica-se que, em ambos os momentos, analisando as estatísticas de item-total, o valor do alfa de Cronbach não aumentaria caso qualquer item fosse excluído. Relativamente às correlações item-total corrigido, os valores para o primeiro e segundo momentos são superiores a 0,30, verificando-se uma elevação dos valores do momento inicial para o segundo momento.

Quadro 2 - Estatísticas de Item e Item-Total para a Escala de Expetativas Positivas sobre Fumar, no $1^{\circ}$ Momento e no $2^{\circ}$ Momento. Coimbra, Portugal, 2021 (continua)

\begin{tabular}{|l|l|c|c|c|c|}
\hline \multicolumn{2}{|l|}{} & \multicolumn{2}{|c|}{ Estatísticas de Item } & \multicolumn{2}{c|}{ Estatísticas de Item-Total } \\
\hline Avaliação & Item & Média & $\begin{array}{c}\text { Desvio- } \\
\text { padrão }\end{array}$ & $\begin{array}{c}\text { Correlação } \\
\text { de item total } \\
\text { corrigida }\end{array}$ & $\begin{array}{c}\text { Alfa de } \\
\text { Cronbach se o } \\
\text { item for excluído }\end{array}$ \\
\hline \multirow{2}{*}{$1^{\circ}$ Momento } & Item 1 & 1,33 & 0,591 & 0,39 & 0,531 \\
\cline { 2 - 6 } & Item 2 & 1,78 & 0,883 & 0,454 & 0,433 \\
\cline { 2 - 6 } & Item 3 & 1,45 & 0,719 & 0,402 & 0,497 \\
\cline { 2 - 6 } & Alfa de Cronbach Total = 0,595 & \multicolumn{3}{|l}{} \\
\cline { 2 - 6 } &
\end{tabular}




\begin{tabular}{|l|l|c|c|c|c|}
\hline \multirow{3}{*}{$2^{\circ}$ Momento } & Item 1 & 1,3 & 0,639 & 0,511 & 0,567 \\
\cline { 2 - 5 } & Item 2 & 1,7 & 0,872 & 0,524 & 0,533 \\
\cline { 2 - 5 } & Item 3 & 1,43 & 0,783 & 0,448 & 0,627 \\
\cline { 2 - 5 } & \multicolumn{4}{l}{ Alfa de Cronbach Total $=0,673$} & \\
\hline
\end{tabular}

Fonte: Autores (2021)

A Escala de Expectativas Negativas sobre Fumar apresentou, no primeiro momento, um alfa de Cronbach de 0,683 com a média dos itens entre 3,53 (item cinco) e 3,76 (item seis). No segundo momento, o alfa de Cronbach foi de 0,905, variando a média dos itens entre 3,58 (item um e dois) e 3,75 (item três). A maioria respondeu "concordo fortemente" ao item "Fumar prejudica a nossa saúde" (item três). À semelhança da subescala relativa às expectativas positivas, também nesta não seria benéfico excluir qualquer um dos itens (Quadro 3). No que diz respeito às correlações item-total corrigido, os valores são elevados.

Quadro 3 - Estatísticas de Item e Item-Total para a Escala de Expetativas Negativas sobre Fumar, no $1^{\circ}$ Momento e no $2^{\circ}$ Momento. Coimbra, Portugal, 2021

\begin{tabular}{|l|l|c|c|c|c|}
\hline \multicolumn{2}{|c|}{} & \multicolumn{2}{|c|}{ Estatísticas de Item } & \multicolumn{2}{c|}{ Estatísticas de Item-Total } \\
\hline Avaliação & Item & Média & $\begin{array}{c}\text { Desvio- } \\
\text { padrão }\end{array}$ & $\begin{array}{c}\text { Correlação } \\
\text { de item total } \\
\text { corrigida }\end{array}$ & $\begin{array}{c}\text { Alfa de Cronbach } \\
\text { se o item for } \\
\text { excluído }\end{array}$ \\
\hline \multirow{3}{*}{$1^{\circ}$ Momento } & Item 1 & 3,59 & 0,653 & 0,489 & 0,604 \\
\cline { 2 - 6 } & Item 2 & 3,53 & 0,749 & 0,526 & 0,551 \\
\cline { 2 - 6 } & Item 3 & 3,76 & 0,753 & 0,482 & 0,611 \\
\cline { 2 - 6 } & Alfa de Cronbach Total $=0,683$ & & 0,779 & 0,893 \\
\hline \multirow{2}{*}{$2^{\circ}$ Momento } & Item 1 & 3,58 & 0,874 & 0,825 & 0,852 \\
\cline { 2 - 6 } & Item 2 & 3,58 & 0,849 & 0,835 & 0,848 \\
\cline { 2 - 6 } & Item 3 & 3,75 & 0,78 & & \\
\cline { 2 - 6 } & Alfa de Cronbach Total =0,905 & & & \\
\hline
\end{tabular}

Fonte: Autores (2021)

O instrumento Escala de Autoeficácia Antitabaco apresentou, no primeiro momento, um alfa de Cronbach de 0,856, estando a média dos itens entre 3,26 (item seis) e 3,94 (item oito), o que significa que a maioria respondeu talvez possa (codificado com "três") e posso fazer (codificado com "quatro"). No segundo momento, o alfa de Cronbach foi de 0,890, com a média dos itens entre 3,39 (item sete) e 3,93 (item um). Em ambos os momentos, o alfa de Cronbach seria mais elevado caso se excluíssem os itens quatro, cinco e sete no pré-teste e o item sete no pós-teste (Quadro 4). Relativamente às correlações de itemtotal corrigido, apenas um item (item cinco) apresenta uma correlação inferior a 0,3, e tal verifica-se apenas no primeiro momento. 
Quadro 4 - Estatísticas de Item e Item-Total para a Escala de Autoeficácia Antitabaco, no $1^{\circ}$ Momento e no $2^{\circ}$ Momento. Coimbra, Portugal, 2021

\begin{tabular}{|c|c|c|c|c|c|}
\hline \multirow{2}{*}{ Avaliação } & \multirow[b]{2}{*}{ Item } & \multicolumn{2}{|c|}{ Estatísticas de ltem } & \multicolumn{2}{|c|}{ Estatísticas de Item-Total } \\
\hline & & Média & $\begin{array}{l}\text { Desvio- } \\
\text { padrão }\end{array}$ & $\begin{array}{c}\text { Correlação } \\
\text { de item total } \\
\text { corrigida }\end{array}$ & $\begin{array}{c}\text { Alfa de Cronbach } \\
\text { se o item for } \\
\text { excluído }\end{array}$ \\
\hline \multirow{16}{*}{$1^{\circ}$ Momento } & Item 1 & 3,84 & 0,605 & 0,358 & 0,854 \\
\hline & Item 2 & 3,81 & 0,621 & 0,392 & 0,852 \\
\hline & Item 3 & 3,82 & 0,662 & 0,41 & 0,852 \\
\hline & Item 4 & 3,65 & 0,915 & 0,328 & 0,857 \\
\hline & Item 5 & 3,69 & 0,883 & 0,293 & 0,859 \\
\hline & Item 6 & 3,26 & 0,887 & 0,521 & 0,846 \\
\hline & Item 7 & 3,4 & 0,874 & 0,309 & 0,858 \\
\hline & Item 8 & 3,94 & 0,385 & 0,542 & 0,85 \\
\hline & Item 9 & 3,42 & 0,978 & 0,555 & 0,844 \\
\hline & Item 10 & 3,43 & 0,948 & 0,672 & 0,836 \\
\hline & Item 11 & 3,5 & 0,888 & 0,722 & 0,834 \\
\hline & Item 12 & 3,41 & 0,954 & 0,677 & 0,836 \\
\hline & Item 13 & 3,69 & 0,624 & 0,525 & 0,847 \\
\hline & Item 14 & 3,53 & 0,774 & 0,616 & 0,841 \\
\hline & Item 15 & 3,45 & 0,836 & 0,587 & 0,842 \\
\hline & \multicolumn{5}{|c|}{ Alfa de Cronbach Total $=0,856$} \\
\hline \multirow{16}{*}{$2^{\circ}$ Momento } & Item 1 & 3,93 & 0,389 & 0,488 & 0,887 \\
\hline & Item 2 & 3,9 & 0,418 & 0,563 & 0,884 \\
\hline & Item 3 & 3,89 & 0,463 & 0,47 & 0,886 \\
\hline & Item 4 & 3,79 & 0,723 & 0,447 & 0,888 \\
\hline & Item 5 & 3,79 & 0,733 & 0,457 & 0,888 \\
\hline & Item 6 & 3,41 & 0,791 & 0,439 & 0,889 \\
\hline & Item 7 & 3,39 & 0,825 & 0,353 & 0,894 \\
\hline & Item 8 & 3,92 & 0,396 & 0,511 & 0,886 \\
\hline & Item 9 & 3,6 & 0,791 & 0,665 & 0,878 \\
\hline & Item 10 & 3,62 & 0,751 & 0,767 & 0,873 \\
\hline & Item 11 & 3,68 & 0,71 & 0,723 & 0,875 \\
\hline & Item 12 & 3,65 & 0,755 & 0,761 & 0,873 \\
\hline & Item 13 & 3,75 & 0,589 & 0,606 & 0,881 \\
\hline & Item 14 & 3,73 & 0,665 & 0,66 & 0,879 \\
\hline & Item 15 & 3,61 & 0,683 & 0,647 & 0,879 \\
\hline & \multicolumn{5}{|c|}{ Alfa de Cronbach Total $=0,890$} \\
\hline
\end{tabular}

Fonte: Autores (2021) 
Um dos motivos que levou à opção pela tradução e adaptação dos instrumentos de avaliação apresentados foi a inexistência de escalas desenvolvidas em Portugal ou traduzidas e validadas para a população portuguesa, específica dos adolescentes, que permitissem a avaliação dos constructos associados à literacia em saúde relacionada com o tabaco.

Como referido, nas versões traduzidas dos instrumentos foram removidos os itens que constavam nas versões originais relativos ao snus, um produto derivado do tabaco, sem combustão, para uso oral. À exceção de alguns países nórdicos, onde o consumo desse produto ultrapassa o consumo de cigarros, no resto do mundo as prevalências de seu consumo são baixas ${ }^{(10)}$. Em Portugal, não estão documentados dados específicos acerca do snus, uma vez que vários relatórios utilizam apenas a designação geral de tabaco(11) para caracterizar as prevalências, podendo estes produtos estar incluídos no consumo de tabaco geral. Assim, considerou-se que, não tendo sido encontrada evidência científica relativa à prevalência do consumo de snus em Portugal que justificasse a abordagem e intervenção com foco também nesta substância, seria o uso de tabaco referente apenas ao consumo de cigarros que seria integrado nos instrumentos de avaliação.

A consistência interna representa a média das correlações entre os itens de um instrumento(9), pelo que apenas escalas com mais de um item permitem a avaliação desta propriedade. Assim, não foi possível avaliar a consistência interna dos instrumentos Atitudes face ao Uso de Tabaco e Motivação para Recusar Utilização de Tabaco no Futuro, pois as versões traduzidas ficaram constituídas por apenas um item cada, o que pode dificultar a avaliação da qualidade do instrumento. No entanto, o objetivo destes instrumentos é avaliar alguns fatores que influenciam a literacia em saúde relacionada com o tabaco, e as questões que integram o instrumento são simples e dirigidas, derivadas de um estudo que procurou, precisamente, identificar os determinantes da literacia em saúde relacionada com as questões do tabaco ${ }^{(6)}$, pelo que se considera que estas avaliam o que é pretendido.

No que diz respeito à consistência interna dos instrumentos Motivos para Uso de Tabaco, Escala de Expectativas sobre Fumar (Escala de Expectativas Positivas sobre Fumar e Escala de Expectativas Negativas sobre Fumar) e Escala de Autoeficácia Antitabaco, todos apresentaram um alfa de Cronbach mais alto no primeiro momento quando comparado com o segundo momento.

Relativamente à escala Motivos para Uso de Tabaco, em comparação com os resultados obtidos por Parisod ${ }^{(4)}$, relativos às versões finlandesas dos instrumentos, 0 alfa de Cronbach foi mais baixo tanto no primeiro $(\alpha=0,85)$ como no segundo momento $(\alpha=0,86)$. No entanto, verificou-se um aumento do alfa do primeiro para o segundo momento em ambos os estudos, tendo este sido maior no estudo da versão traduzida do instrumento. A versão original apresentou uma consistência interna considerada boa ${ }^{(8)}$, tendo o instrumento traduzido apresentado uma consistência interna razoável no primeiro momento e boa no segundo.

A Escala de Expectativas Positivas sobre Fumar apresentou valores de alfa de Cronbach que apontam para uma consistência interna fraca ${ }^{(8)}$. Também Parisod ${ }^{(4)}$ encontrou valores que apontam para uma consistência interna fraca no primeiro momento $(a=0,59)$ e aceitável no segundo $(a=0,71)$. Verifica-se, pois, que estes valores do alfa, em comparação com o resultado da validação da versão original da escala $(a=0,78)$, são mais baixos, apresentando a versão desenvolvida em Taiwan melhor consistência interna do que a versão finlandesa e a versão em português europeu, com uma consistência interna razoáve|(5,8), tendo considerado que um alfa de Cronbach superior a 0,7 correspondia a uma consistência interna razoável.

Considera-se que a Escala de Expectativas Negativas sobre Fumar apresentou uma 
consistência interna fraca no primeiro momento, aumentando de forma significativa no segundo, sendo considerada como muito boa. A versão validada na Finlândia(4) apresentou um alfa de Cronbach de 0,71 no pré-teste e de 0,87 no pós-teste, tendo assim variado a consistência interna entre razoável e boa. Relativamente à versão original do instrumento ${ }^{(5)}$, os resultados da validação revelaram um alfa de Cronbach de 0,76 e, portanto, uma consistência interna razoável|(8).

A Escala de Autoeficácia Antitabaco apresentou uma consistência interna boa em ambos os momentos ${ }^{(8)}$. A versão de Parisod(4) apresentou um alfa de Cronbach de 0,89 no primeiro momento e de 0,91 no segundo, ou seja, consistência interna boa e muito boa, respectivamente. Também a versão original apresentou uma consistência interna boa, segundo a classificação adotada por Chen ${ }^{(5)}$, e muito boa de acordo com Pestana e Gageiro ${ }^{(8)}$, apresentando um alfa de 0,90. De todas as versões, as validadas na Turquia(12) foram as que apresentaram melhor consistência interna (ASSES: $a=0,93$; POS-SOES: $a=0,85$; NEG-SOES: 0,91).

Podem considerar-se como limitações deste estudo a sua realização numa região específica de Portugal, o que não permite generalização dos resultados, e o tamanho da amostra, que pode ser considerado reduzido. Assim, recomenda-se a realização de mais estudos para testar a validade dos instrumentos, tendo em vista a importância que estes assumem em um processo de investigação.

\section{CONCLUSÃO}

Desta tradução e adaptação transcultural para português europeu, resultaram os itens Atitudes face ao Uso de Tabaco e Motivação para Recusar a Utilização de Tabaco no Futuro e as escalas Motivos para Uso de Tabaco, Escala de Expetativas sobre Fumar e Escala de Autoeficácia Antitabaco.

$\mathrm{Na}$ validação preliminar, as versões traduzidas revelaram ser compatíveis com a análise dos constructos a que se propuseram, ao apresentarem valores de consistência interna boa a muito boa no segundo momento de avaliação, com a exceção da escala que avaliou as expectativas positivas, que revelou valores inferiores, no entanto, superiores a 0,65. Recomenda-se, tendo em conta a importância que os instrumentos de avaliação assumem num processo de investigação, que mais estudos sejam realizados para perceber quais os melhores instrumentos para a avaliação da literacia em saúde relacionada com as questões do tabaco de adolescentes.

Não tendo sido encontrados instrumentos de avaliação adaptados para a população portuguesa, especificamente dos adolescentes, a tradução e adaptação destes instrumentos apresenta-se como relevante, pois constitui a primeira contribuição para a existência de instrumentos específicos para a avaliação da literacia em saúde acerca do tabaco, em português europeu, dirigidos a adolesçentes.

\section{REFERÊNCIAS}

1. Bröder J, Okan O, Bauer U, Bruland D, Schlupp S, Bollweg TM, et al. Health literacy in childhood and youth: a systematic review of definitions and models. BMC Public Health. [Internet]. 2017 [acesso em 14 abr 2021]; 17(1). Disponível em: http://doi.org/10.1186/s12889-017-4267-y. 
3. Parisod H, Pakarinen A, Axelin A, Danielsson-Ojala R, Smed J, Salanterä S. Designing a health-game intervention supporting Health Literacy and a Tobacco-Free Life in Early Adolescence. Games Health J [Internet]. 2017 [acesso em 14 abr 2021]; 6(4). Disponível em: http://doi.org/10.1089/g4h.2016.0107.

4. Parisod H, Pakarinen A, Axelin A, Löyttyniemi E, Smed J, Salanterä S. Feasibility of mobile health game "Fume" in supporting tobacco-related health literacy among early adolescents: a three-armed cluster randomized design. Int. J. Med. Inform. [Internet]. 2018 [acesso em 14 abr 2021]; 113. Disponível em: http://doi.org/10.1016/j.ijmedinf.2018.02.013.

5. Chen CJ, Yeh MC, Tang FI, Yu S. The smoking outcome expectation scale and anti-smoking self-efficacy scale for early adolescents: instrument development and validation. J Sch Nurs [Internet]. 2015 [acesso em 14 abr 2021]; 31(5). Disponível em: http://doi.org/10.1177/1059840514560352.

6. Parisod H, Axelin A, Smed J, Salanterä S. Determinants of tobacco-related health literacy: a qualitative study with early adolescents. Int J Nurs Stud. [Internet]. 2016 [acesso em 14 abr 2021]; 62. Disponível em: http://doi.org/10.1016/j.jinurstu.2016.07.012.

7. Beaton D, Bombardier C, Guillemin F, Ferraz MB. Recommendations for the cross-cultural adaptation of the DASH \& QuickDASH outcome measures contributors to this document. Inst. Work Health. [Internet]. 2007 [acesso em 14 abr 2021]. Disponível em: https://www.researchgate.net/publication/265000941 Recommendations for the Cross-Cultural Adaptation of the DASH QuickDASH Outcome Measures Contributors to this Document/link/53fdd6140cf22f21c2f85143/download.

8. Pestana MH, Gageiro JN. Análise de dados para ciências sociais: a complementariedade do SPSS. 6. ed. Lisboa: Edições Sílabo; 2014.

9. Streiner DL, Norman GR, Cairney J. Health measurement scales: a practical guide to their development and use. 5. ed. Inglaterra: Oxford University Press; 2015.

10. Zhu SH, Gamst A, Lee M, Cummins S, Yin L, Zoref L. The use and perception of electronic cigarettes and snus among the U.S. Population. PLoS ONE. [Internet]. 2013 [acesso em 14 abr 2021]; 8(10). Disponível em: http://doi.org/10.1371/journal.pone.0079332.

11. Balsa C, Vital C, Urbano C, Serviço de intervenção nos comportamentos aditivos e nas dependências. IV Inquérito Nacional ao Consumo de Substâncias Psicoativas na População Geral, Portugal 2016/17. [Internet]. Portugal: SICAD; 2018 [acesso em 14 abr 2021]. Disponível em: http://www.sicad.pt/ PT/Estatisticalnvestigacao/EstudosConcluidos/Paginas/detalhe.aspx?itemld=181\&lista=SICAD ESTUDOS\&bkUrl=/BK/Estatisticalnvestigacao/EstudosConcluidos.

12. Bektas I, Bektas M, Ayar D, Kudubeş AA, Selekoglu Y, Sal Altan S, et al. The psychometric properties of the Turkish version of the smoking outcome expectation scale and the anti-smoking self-efficacy scale for early adolescents. J Subst Use. [Internet]. 2017 [acesso em 14 abr 2021]; 22(6). Disponível em: http:// doi.org/10.1080/14659891.2017.1296042.

\section{INSTRUMENTOS PARA EVALUAR LA ALFABETIZACIÓN EN SALUD SOBRE EL TABACO: TRADUCCIÓN Y ADAPTACIÓN AL PORTUGUÉS EUROPEO}

\section{RESUMEN:}

Objetivo: traducir y adaptar culturalmente al portugués europeo los instrumentos de evaluación de la alfabetización en salud sobre el tabaco Attitudes Towards Tobacco Use, Tobacco-Use Motives y Motivation to Decline Tobacco Use in the Future, y Smoking Outcome Expectation Scale y Anti-Smoking Self-Efficacy Scale, y realizar una validación preliminar. Método: traducción y adaptación transcultural según las recomendaciones del Institute for Work and Health y validación preliminar en 144 adolescentes de dos escuelas públicas de la región central de Portugal, en abril y mayo de 2019. Resultados: se eliminaron los artículos relacionados con la snus, ya que no eran relevantes en el contexto portugués. De este modo, dos instrumentos se componen de un solo ítem. Los valores del alfa de Cronbach de las versiones en portugués europeo fueron 0,799, 0,673, 0,905 y 0,890. Conclusión: contribuye a la existencia de instrumentos de evaluación de la alfabetización en salud sobre el tabaco, adaptados al portugués europeo, para la población adolescente.

DESCRIPTORES: Estudio de Validación; Escalas; Alfabetización en Salud; Tabaco; Adolescentes. 


\section{INSTRUMENTOS DE AVALIAÇÃO DA LITERACIA EM SAÚDE ACERCA DO TABACO: TRADUÇÃO E ADAPTAÇÃO PARA PORTUGUÊS EUROPEU}

\section{RESUMO:}

Objetivo: traduzir e adaptar culturalmente para português europeu os instrumentos de avaliação da literacia em saúde acerca do tabaco Attitudes Towards Tobacco Use, Tobacco-Use Motives e Motivation to Decline Tobacco Use in the Future, e Smoking Outcome Expectation Scale e Anti-Smoking Self-Efficacy Scale, e realizar validação preliminar. Método: tradução e adaptação transcultural de acordo com as recomendações do Institute for Work and Health e validação preliminar em 144 adolescentes de duas escolas públicas da região central de Portugal, em abril e maio de 2019. Resultados: foram retirados itens relativos a snus, por não apresentarem relevância no contexto português. Assim, dois instrumentos passaram a ser constituídos apenas por um item. Os valores de alfa de Cronbach das versões em português europeu foram 0,799, 0,673, 0,905 e 0,890. Conclusão: contribui para a existência de instrumentos de avaliação da literacia em saúde acerca do tabaco, adaptados para português europeu, para a população dos adolescentes.

DESCRITORES: Estudo de Validação; Escalas; Letramento em Saúde; Tabaco; Adolescentes.

*Artigo extraído da dissertação de mestrado "Efeito da versão portuguesa do jogo de saúde Fume na literacia em saúde acerca do tabaco de adolescentes". Escola Superior de Enfermagem de Coimbra, 2020.

Recebido em: 12/05/2021

Aprovado em: 12/07/2021

Editora associada: Luciana Puchalski Kalinke

Autor Correspondente:

Daniela Lourenço Pinto

Unidade de Investigação em Ciências da Saúde: Enfermagem, Escola Superior de Enfermagem de Coimbra - Coimbra, Portugal

E-mail: danielapinto@esenfc.pt

Contribuição dos autores:

Contribuições substanciais para a concepção ou desenho do estudo; ou a aquisição, análise ou interpretação de dados do estudo - Pinto DL, Parisod H, Nyman J, Barroso TMMD de A; Elaboração e revisão crítica do conteúdo intelectual do estudo - Pinto DL, Barroso TMMD de A; Responsável por todos os aspectos do estudo, assegurando as questões de precisão ou integridade de qualquer parte do estudo - Pinto DL, Barroso TMMD de A. Todos os autores aprovaram a versão final do texto.

Copyright (? 2021 Este é um artigo em acesso aberto distribuído nos termos da Licença Creative Commons Atribuição, que permite o uso irrestrito, a distribuição e reprodução em qualquer meio desde que o artigo original seja devidamente citado. 